\title{
Looking Beyond the Indication: Bone Scan for Trauma Providing Unsuspected Initial Diagnosis of Acute Critical Lower Limb Arterial Compromise
}

\author{
Fathima Fijula Palot Manzil ${ }^{1}$, Samuel E. Almodovar ${ }^{2}$, and Pradeep G. Bhambhvani ${ }^{3}$ \\ ${ }^{1}$ Clinical Radiology, Weill Cornell Medical College, Qatar, and Department of Radiology, Hamad General Hospital, Doha, Qatar; \\ ${ }^{2}$ Department of Radiology, Orlando VA Medical Center, Orlando, Florida; and ${ }^{3}$ Division of Molecular Imaging and Therapeutics, \\ Department of Radiology, University of Alabama at Birmingham, Birmingham, Alabama
}

We report a case of rare incidental initial detection of unsuspected arterial supply compromise to the right lower extremity on a bone scan obtained to rule out fracture in a patient with right hip pain after a fall. Follow-up CT angiogram confirmed near-complete occlusion of right lower extremity arteries for which patient underwent above-knee amputation.

Key Words: three phase bone scan; ischemia; CT angiogram; amputation

J Nucl Med Technol 2018; 46:67-68

DOI: $10.2967 /$ jnmt.117.200618

$\mathbf{T}$

hree-phase bone scintigraphy is a useful tool to assess arterial flow, relative soft-tissue perfusion, and variations in bone turnover. Systematic evaluation of all 3 phases is crucial to detect skeletal and unsuspected extraskeletal abnormalities.

\section{CASE REPORT}

A 101-y-old patient with a history of atrial fibrillation presented to our hospital with right hip pain and difficulty walking after a fall. A plain CT scan of the bony pelvis and 3-phase ${ }^{99 \mathrm{~m}} \mathrm{Tc}$-methylene diphosphonate bone scintigraphy performed to rule out clinically suspected occult hip bone injury were negative for fracture. However, scintigraphy showed near-absent radiotracer activity within the right lower limb arteries on the flow phase (Fig. 1A), decreased soft-tissue uptake around the right knee and lower leg on the blood-pool phase (Fig. 1B), and decreased radiotracer uptake in the right femur, especially distally, and

Received Aug. 10, 2017; revision accepted Aug. 28, 2017.

For correspondence or reprints contact: Fathima Fijula Palot Manzil, Department of Clinical Radiology, Weill Cornell Medical College, Qatar, and Department of Radiology, P.O. Box 3050, Hamad General Hospital, Doha, Qatar.

E-mail: drfijulasurjith@yahoo.com

Published online Nov. 10, 2017.

COPYRIGHT (C) 2018 by the Society of Nuclear Medicine and Molecular Imaging. the lower leg bones on the delayed skeletal phase (Fig. 1C). Follow-up urgent CT angiogram on the same day demonstrated extensive thromboembolism of right lower limb arteries (Fig. 2). The right leg turned extremely painful and cold after a day. On physical examination, there was no palpable or audible right femoral, popliteal, and pedal flow. The patient had limited right knee flexion and limited sensation from the knee downward and could not move the right foot, and underwent right transfemoral above-knee amputation.

\section{DISCUSSION}

Major factors determining radiotracer uptake in 3-phase bone scintigraphy are local blood flow, extraction efficiency, and osteoblastic activity. Impaired circulation,

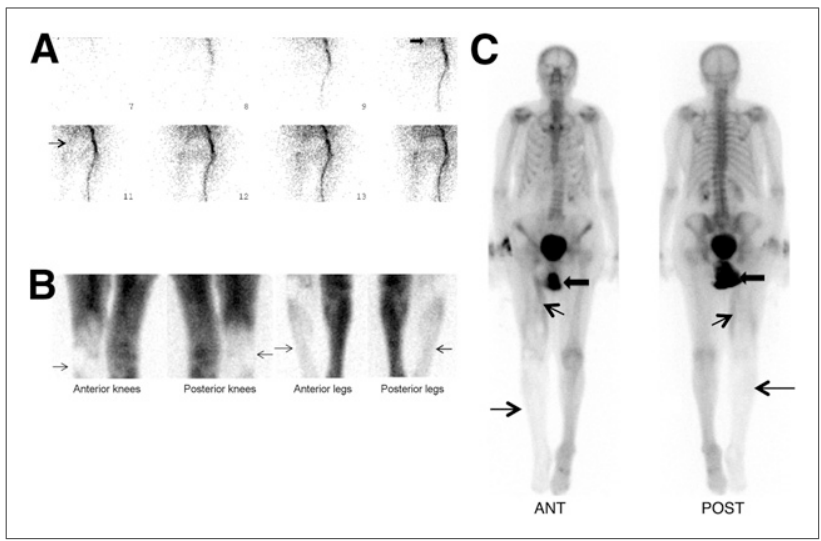

FIGURE 1. (A) Blood-flow images of 3-phase bone scintigraphy of thighs in anterior projection shows near-absent radiotracer uptake within right common iliac, external iliac (thick arrow), and proximal superficial femoral arteries (thin arrow). (B) Blood-pool images at knee/leg level in anterior and posterior projections show decreased soft-tissue uptake in right knee and leg (arrows). (C) Delayed whole-body bone scan in anterior and posterior projections show decreased radiotracer uptake in right femur, especially distally, and bones of right lower leg (thin transverse arrows). Increased linear uptake at medial right thigh (oblique arrows) possibly represents muscle injury. Note urinary activity in diaper (thick arrows). 


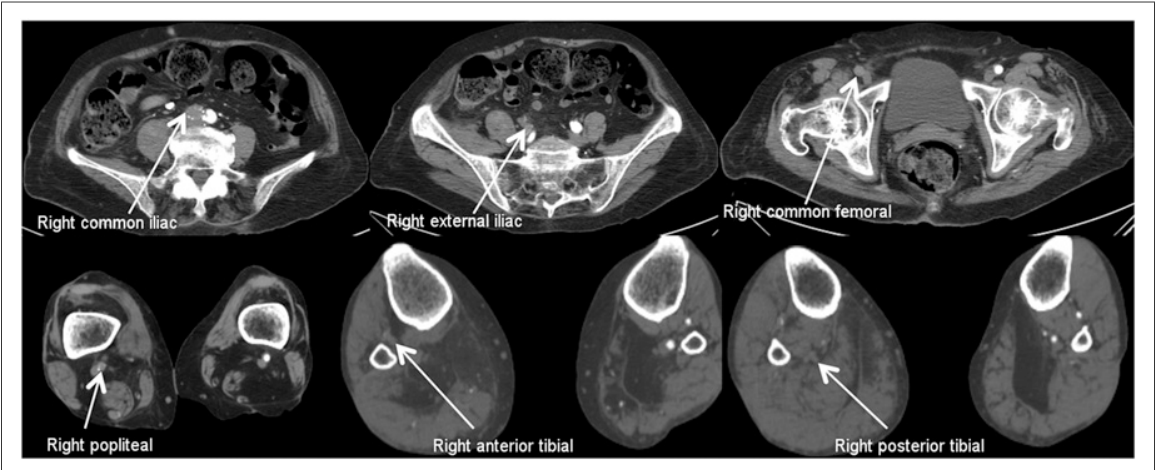

FIGURE 2. CT angiogram of pelvis/lower limbs demonstrates extensive thromboembolism of right common iliac, external iliac, common femoral, popliteal, anterior, and posterior tibial arteries (white arrows).

hydration level, renal function, and age also influence uptake $(1,2)$. A bone scan is a useful adjunct to choose appropriate amputation level by aiding in distinguishing viable from nonviable tissue (3).

Assessing symmetry of radiotracer distribution is crucial. Venous or lymphatic obstruction and inflammation can cause asymmetrically increased tracer uptake. Unilateral paralysis can reduce ipsilateral uptake with increased contralateral activity from compensatory overuse. Decreased radiotracer activity can be seen in compromised arterial flow.

The functional status of the limbs could be improved with adequate collateral flow (4). However, because of minimal time available for collateral development in cases of acute thromboembolism, chances for above-knee amputation are doubled compared with chronic ischemia (5).

\section{CONCLUSION}

While interpreting bone scans, it is important not to confine our attention only to the detection of skeletal abnormalities. In our case, bone scan led to the initial diagnosis of critical ischemia of lower limb and apparent necrosis of thigh muscles, which led to further investigations, definitive diagnosis, and treatment. Any asymmetry in radiotracer uptake pattern in all phases on a 3-phase bone scan should not be overlooked.

\section{DISCLOSURE}

No potential conflict of interest relevant to this article was reported.

\section{REFERENCES}

1. Medina-Gálvez N, Pedraz T. Nuclear medicine in musculoskeletal disorders: clinical approach. In: Gholamrezanezhad A, ed. 12 Chapters on Nuclear Medicine. Rijeka, Croatia: InTech; 2011:97-135.

2. Love C, Din AS, Tomas MB, et al. Radionuclide bone imaging: an illustrative review. Radiographics. 2003;23:341-358.

3. Masaoka S. Evaluation of arterial obstructive leg and foot disease by three-phase bone scintigraphy. Ann Nucl Med. 2001;15:281-287.

4. Weitz JI, Byrne J, Clagett GP, et al. Diagnosis and treatment of chronic arterial insufficiency of the lower extremities: a critical review. Circulation. 1996;94:3026-3049.

5. Campbell WB, Marriott S, Eve R, et al. Amputation for acute ischaemia is associated with increased comorbidity and higher amputation level. Cardiovasc Surg. 2003;11:121-123. 